Theological Research • volume 2 (20I4) NUMber I • P. 5-I7 DoI: http://dx.doi.org/Io.15633/thr.675

JeAn-Louis Bruguès, O.P.

Archivist and Librarian of Holy Roman Church

\title{
Ethique et Politique
}

\section{Abstract: Ethics and Politics}

The lecture engages the debate concerning the relation between politics and ethics. Ethics is the guardian of good practice and policy the guardian of the quality of life in the community. The author discusses their mutual historical development paying special attention to two dates. First in 1789, when the Bastille was demolished and the Republic founded with a slogan of ethical content ("Liberty, Equality, Fraternity"), but only in order to control it. The second date is 1989 , the year in which the Berlin Wall fell, constitutes a turning point and the dominance of ethics over politics promoted by the triad: democracy, accountability, and human rights, which undoubtedly belong to the highest value. The problem with this is that ethical values can be used in an ideological way. In the final section, the Christian position on the proper relationship between ethics and policy and vice versa is discussed.

\section{KeYWORDS}

ethics, politics, political correctness, procedural ethics 


\section{INTRODUCTION}

On racontait chez les dominicains de mon pays une histoire dont je ne suis pas sûr qu'elle fût une simple légende. Si au cours de vos études de théologie, vous vous étiez révélé un élève particulièrement doué, on vous proposait d'enseigner la matière royale par excellence, la dogmatique. Si vous aviez été seulement un bon étudiant, vous risquiez d'enseigner la philosophie ou l'Ecriture sainte. Si vous aviez été un étudiant un peu au-dessus de la moyenne, les portes de l'histoire de l'Eglise ou de la liturgie pouvaient vous être ouvertes. Si enfin vous aviez passé vos examens avec des notes au ras des pâquerettes, vous pouviez enseigner le droit canon, ou la théologie morale... ou encore devenir l'économe de votre couvent!

J'avais vingt-cinq ans quand je suis entré chez les Dominicains de la province de Toulouse. J'avais alors passé sept années à l'Université, faisant des études de sciences politiques et de sciences économiques, matières pour lesquelles je n'ai jamais cessé de montrer de l'intérêt. Non sans naïveté, j'avais pensé que ce temps passé à l'Université d'Etat pouvait me valoir quelque ristourne dans mes études théologies, des économies de crédits, comme on disait alors. On m'expliqua vite qu'il n'en était pas question, car la théologie représentait une matière vraiment particulière, unique même, qu'il convenait de parcourir de fond en comble. Je n'y fus guère brillant.

C'est ainsi que le provincial me fit appeler un jour, vers le milieu de mes études. On me destinait à l'enseignement de la théologie morale. Un vieux sage du couvent chercha à méclairer : «Quelles sont les activités qui vous procurent le plus de plaisir ? ", me demanda-t-il. "Lire des romans et aller au cinéma ", lui ai-je répondu. «Alors, le choix s'impose de lui-même, reprit-il : prenez la théologie morale ». Pourquoi ? Parce que la première attitude exigée chez celui qui se prépare à entrer en théologie morale est la curiosité, m’expliqua-t-il : curiosité envers les choses de la vie et bonne connaissance des méandres du cœur humain. Curiosité pour le métier d'homme. Etre un humaniste, en un mot. Il nétait pas nécessaire de travailler beaucoup d'ouvrages de théologie pour cela : "Les romans et les films suffisaient bien...» Celui qui cherchait à me convaincre de la sorte était lui-même professeur de théologie morale ; il avait été choisi comme expert du pape au concile de Vatican II.

Le Père Labourdette, puisque c'est de lui qu'il s'agissait, ajouta : "Vous entrez dans une matière fort méprisée, mais prenez patience : 
un jour viendra où cette discipline sera enviée des autres! ». Je voyais bien que le vieil enseignant se moquait gentiment de moi. Pourtant, ses propos restèrent gravés dans ma mémoire. Le fait est que la théologie morale était tombée, en France du moins, dans un état de profonde déréliction. Pendant deux années, les séminaristes de Toulouse ne reçurent aucun enseignement dans cette matière réputée ingrate et rébarbative, puisque personne voulait l'enseigner ; on répétait volontiers qu'ils ne s'en portaient pas plus mal. Après mai 68 , comment pouvaiton s'intéresser encore à la chose morale ? Comme jétais le plus jeune, le sort tomba sur moi : on me demanda d'occuper une chaire tombée en quenouilles.

Que de changements depuis lors! Il ne serait pas difficile de montrer que la théologie morale a été cette partie de la théologie qui, au cours des trois dernières décennies, a connu les variations les plus fortes, relatives à son contenu, certes, mais surtout à la manière dont elle a été perçue par l'opinion générale. Ce soir, je chercherai à comprendre avec vous pourquoi son image est passée d'une extrémité à l'autre, en un laps de temps finalement réduit, de la déconsidération à l'exaltation. Soyons plus précis : mon double intérêt pour la chose politique et pour la théologie morale me permit d'assister avec une curiosité aigüe à un revirement de tendances, ou mieux encore à un chassé-croisé, dont il existe peu d'exemples dans l'Histoire moderne. C'est ce que je voudrais raconter ce soir.

Je le ferai en trois parties:

- Le royaume perdu

- Le triomphe du «politiquement correct»

- Le génie du christianisme

\section{Le Royaume Perdu}

L'éthique et la politique, ainsi que le suggèrent l'histoire de ces derniers siècles, ne seraient-elles pas, en effet, comme deux jumelles issues d'un même oeuf, celui de la destinée humaine ? Nées du mariage du bien, le père, et de la nécessité, la mère, elles tiennent évidemment des deux parents. La première, léthique - mais est-ce bien la première, car avec les jumeaux, l'aînesse reste toujours problématique -, l'éthique donc viendrait plutôt du côté du père, que l'on dit un peu rêveur, ou idéaliste, attendant du ciel le signe décisif qui enfin convaincrait les hommes 
de se tenir debout et de s'engager dans les voies de la générosité. On a parfois défini l'éthique comme la science du bien agir. Sa soeur, la politique, a toujours regardé, elle, du côté de sa mère. Comme la nécessité, elle a gardé ses pieds sur la terre et se présente volontiers comme la ménagère de la Cité, soucieuse de mettre de l'ordre là où tant de raisons, tant de passions, tant d'inclinations pousseraient au laisser-aller et à la négligence. La politique est la gardienne du vivre-ensemble.

En réalité, les deux soeurs tiennent des deux parents. Que serait, en effet, une éthique qui ignorerait les nécessités humaines du coeur et de l'esprit, du corps et de l'âme ? S. Thomas expliquait qu'en morale, il fallait partir comme d'un principe de ce qui se faisait. L'éthique part donc des moeurs, et c'est pour cela que l'on peut aussi bien l'appeler morale. Que serait, par ailleurs, une politique qui ne garderait pas un oeil sur l'utopie et, s'employant au bien-être des citoyens, ne chercherait à les éveiller aux devoirs du bien vivre-ensemble ? La politique aussi se prend à rêver, comme son père, à quelque "règne de justice et de paix ", pour parler comme les psaumes...

Or donc, l'éthique et la politique se partagent un même héritage, un commun domaine familial : l'agir des hommes.

On peut être jumeaux, et jumeaux zygotes, et rêver aussi à d'autres relations. Vint un moment où nos soeurs cherchèrent ailleurs que dans le giron familial à contracter de nouvelles alliances. Longtemps, l'éthique regarda du côté de la religion. Elle crut pouvoir s'appuyer sur son antique sagesse pour fonder et justifier les normes et les principes dont les hommes avaient besoin pour guider leur existence; puis, à une date récente, avec Kant exactement, elle choisit son autonomie et décida de ne s'appuyer que sur elle-même. Longtemps la politique convola avec la philosophie : Platon ne voulait-il pas confier le gouvernement de la Cité à des philosophes qui, parce qu'ils avaient réussi à s'élever jusqu'à l'empirée des idées pures, ne pouvaient que régner avec la plus grande prudence ? Puis, les fruits récoltés de cette alliance, je veux parler des idéologies, lui parurent trop verts; la politique s'affranchit à son tour de la philosophie et crut pouvoir se conduire par elle-même.

Nos deux soeurs se retrouvèrent donc seules, condamnées à occuper un même espace forcément étroit. Leur « convivence " connut des saisons. Il y eut des conflits et des guerres ouvertes, suivies de longues accalmies. Dans les familles bien-pensantes, on ne se sépare pas ; mais les paix restaient armées. Les jumelles prétendirent se respecter toujours; en réalité, leur rivalité ne faiblit jamais. C'est une histoire aussi vieille 
que le monde : la Bible en parle d'abondance avec Caïn et Abel, Esaü et Jacob, Joseph et ses frères. Sous le couvert d'une mutuelle estime, chacune rêvait de supplanter sa jumelle.

Dans cette histoire familiale, la nôtre en somme, deux dates méritent de retenir notre attention, me semble-t-il, deux dates séparées par deux siècles exactement. I789, 1989: il y a dans l'exactitude de cet espacement une raison supplémentaire qui donne à penser.

I789: la France était grosse de la République et déjà, avec la prise de la Bastille, l'enfant lui donnait des coups de pied au ventre. On ne mesure peut-être plus aujourd'hui la nouveauté radicale de l'événement. Lassés de millénaires tutelles, les peuples aspiraient à un monde nouveau, plus nouveau encore que ces Etats-Unis d'Amérique nés quelques années auparavant. Il s'agissait de bâtir une société comme on n'en n'avait jamais conçue, mais que pourtant avaient pressentie ces grands visionnaires que furent Locke et Montesquieu. Il s'agissait en somme de dépasser les rêves les plus fous, car se contenter de les réaliser aurait semblé trop simple et trop commun. L'événement devenait un avènement. Bref, la politique crut enfin l'emporter définitivement sur son éternelle rivale. Elle imagina une de ces ruses géniales comme les aimait Hegel.

Le premier acte de la jeune République s'apparentait à un réflexe d'apparence aristocratique. Elle se donna une devise. Comme dans les familles bien nées, elle voulut se reconnaître et se faire reconnaître surtout en un emblème, version moderne des blasons de jadis. Sa trouvaille se présentait au premier abord comme un hommage à l'éthique, sa soeur : liberté, égalité, fraternité. Aucun de ces concepts nétait de nature politique. La République voulut rassembler en eux le meilleur de cet immense effort des hommes de bonne volonté au travers des millénaires, pour témoigner du bien. Elle se fit l'héritière d'Athènes, avec la liberté, de Rome, avec l'égalité, et de Jérusalem, avec la fraternité.

Le génie de la ruse consistait en ceci : sous le couvert de rendre un hommage appuyé à l'éthique, la politique lui signifiait son congé. L'hommage dissimulait un adieu, ou plus exactement un double adieu : au passé d'abord, puisque l'humanité entrait dans une ère nouvelle, à l'éthique ensuite et surtout qui, trop flattée, ne mesura point sur le moment sa profonde déchéance. A partir de 1789 , la politique occupa tous les domaines de l'agir humain et prétendit y régner sans partage. 
Durant deux siècles, la politique s'employa à convertir la liberté en libertés publiques. Personne ne songerait à nier les réels progrès en humanité qu'apporta l'éclosion de ces libertés, mais dans le passage du singulier au pluriel, la politique s'octroyait le droit de réglementer, de limiter, voire de supprimer, selon la nécessité - toujours elle ! -, ce qui par nature ne supportait aucune contrainte.

Sous le prétexte de faire régner l'égalité entre les individus et entre les peuples, la politique de ces siècles despotes, selon la juste expression du poète Ossip Mandelstam, imposa le principe absolu de la loi du plus fort : loi d'airain du marché, tandis qu'une improbable « main invisible " devait rétablir l'harmonie des intérêts, loi plus rude encore des nationalités qui provoqua sur notre continent les guerres les plus sanglantes de tous les temps; loi de la realpolitik chère au chancelier Bismarck et déjà théorisée par Machiavel et Hobbes, qui sacrifiait sans remords exagérés l'innocence et la vérité sur l'autel de la raison d'Etat ; loi enfin de l'inégalité des races qui édifia Auschwitz ou de l'inégalité des classes qui fit construire les « goulags » de Russie et de Chine.

Je n'ai pas parlé de fraternité, et pour cause : cette valeur éminemment éthique, éminemment chrétienne, ne pouvait s'acclimater à la politique. Il eut fallu évoquer la figure du père pour que les hommes se reconnussent des frères. Or, le père avait été tué en $\mathrm{I} 793$, guillotiné au milieu de son peuple, et sa place ne pouvait que rester vide définitivement. L'Etre suprême, lui, n'éprouvait aucune tendresse paternelle...

Les deux derniers siècles ont ainsi porté la politique à son zénith qui réussit à imposer, en 1968, alors que les révoltes étudiantes menaçaient les pouvoirs en place jusqu'au lointain Mexique, cet étonnant slogan qui a présidé à mes longues études : Tout est politique. Mais déjà la ruine s’annonçait sous le triomphe.

\section{Le Triomphe du « Politiquement Correct »}

L'éthique est une bonne fille et je lui reconnais souvent une candeur coupable. Elle finit toutefois par se morfondre dans les sacristies ou les chaires universitaires de troisième catégorie où l'avait reléguée sa sœur. Jusque pendant les années 70, aucun homme politique n'aurait 
consenti à y faire une référence publique, de peur d'apparaitre vieux jeu ou réactionnaire. "Comment, ça existe encore ? ", me demandait un journaliste quand il apprit que j'enseignais la morale. L'éthique en eut assez d'être traitée en paria de la philosophie, de la théologie et autres sciences humaines. Elle se révolta. Les révoltes des faibles sont parmi les plus terribles.

Léthique aussi se promit de ruser, et le fit d'un manière non moins géniale que sa soeur.

Le mur qui, à Berlin, le 9 novembre 1989 , il y a presque vingt-cinq ans, volait en éclat à la stupéfaction d'un monde ébahi, était celui de la toute-puissance politique qui avait tranché dans le vif des familles, dans le vif d'un pays qui joua un rôle central en Europe, l'Allemagne. Le recul historique nous manque encore pour mesurer l'importance de l'événement. Je formule ici l'hypothèse qu'elle fut décisive et que cet événement marqua le retour offensif de l'éthique.

Depuis quelques années déjà, l'éthique avait oeuvré pour substituer à l'ancienne trilogie une sorte de triangle magique, selon l'expression de Pierre Hassner, constitué par la démocratie, la responsabilité et les droits de l'homme. Cette nouvelle triade dont les termes semblaient de forte teneur politique, puisque le premier était emprunté à la philosophie du même nom (Aristote en parlait déjà) et les deux autres au droit, nétait-elle pas un hommage à la politique ? En réalité, et c'est en cela que consistait sa ruse, l'éthique les fit enfler, gonfler et prendre un tel volume que ces trois concepts finirent par étouffer sa soeur, alors qu'ils étaient censés la valoriser et la régénérer.

Léthique commença par retirer toute espérance à la politique. La force symbolique de ce grand moment historique que représenta la chute du mur de Berlin fut de laisser croire que l'on touchait enfin à la victoire universelle de la démocratie. Si celle-ci était la fin naturelle vers laquelle tendaient, ou devaient tendre tous les régimes politiques, fût-ce à leur corps défendant, comme lorsque les Américains crurent pouvoir l'imposer à des peuples dont il serait ingénu de croire qu'ils y aspiraient tous, en Irak d'abord, puis dans le Moyen Orient, son avènement marquait ce que Fukuyama appelait la fin de l'bistoire. Le meilleur étant advenu, il ne restait plus rien à attendre, sinon d'infinies répétitions. Et c'est ainsi que l'éthique, en portant la démocratie à son extrême, retira à la politique, pour parler comme Ernst Bloch, son principe espérance. L'événement de 1989 permet de comprendre le retournement de l'histoire récente : le passage d'un 
monde ressenti comme culminant et achevé à un monde condamné à la dégénérescence.

Ensuite, l'éthique s'employa à paralyser sa jumelle en conférant à la responsabilité des dimensions proprement extravagantes. Il ne s'agissait plus de la concevoir comme une imputation ou une paternité du sujet envers ses actes - ce qu'elle était depuis les Romains -, mais de lui faire subir un changement d'échelle. Selon le principe formulé par Hans Jonas, nous devrions nous reconnaître responsables de décisions qui engagent désormais, non plus le proche et l'immédiat, mais le très lointain dans le temps et dans l'espace. Ne faut-il pas même demander pardon pour les fautes des siècles qui nous ont précédés? Cette excroissance a conduit au discrédit de l'action politique et à la moralisation excessive du langage : "Plus je me sens responsable, et moins je me sens citoyen. Moins je me sens impliqué dans la vie publique, et plus je cherche en retour à me conduire selon les préceptes d'un code éthique » (Olivier Mongin) : telle pourrait être le leit-motiv qui traverse la littérature morale contemporaine au meilleur de sa forme.

Restait à asséner le coup de grâce. L'éthique se servit des droits de l'h o m me ; elle en fit, non plus un principe éthique, mais un préalable politique. Pas de reconnaissance diplomatique, pas d'aide financière, pas d'échanges commerciaux pour ceux qui n'auraient pas récolté au préalable de bonnes notes dans l'échelle des droits de l'homme : sous la contrainte de l'opinion et la menace d'un nouveau droit d'ingérence, de nombreux Etats se trouvent maintenant réduits à l'impuissance.

Personne ne songerait à nier que la démocratie, la responsabilité et les droits de l'homme ne figurent parmi les plus hautes valeurs de l'esprit. Le danger réside en ce qu'elles se trouvent aujourd'hui récupérées par une idéologie qui prétend faire de la politique en instrumentalisant la morale. L'autonomie du politique : voilà ce qui est en train de s'effacer lentement sous nos yeux. L'idéologie morale conduit à croire que Tout est moral, comme on disait hier : Tout est politique. Contrairement à ce que mes propos laissaient peut-être entendre, la morale contemporaine n'est pas boursouflée par des excès d'humanisme et de confiance. Elle est au contraire blessée par le sentiment d'une profonde fragilité humaine (Olivier Mongin). Elle ne se résigne pas à ce mal radical, irréductible, irrépressible, qui l'oblige à considérer ce qu'il y a d'inhumain dans l'humain. Ce mal, elle le nomme politique. 
Voilà donc le point où je souhaitais me rendre. Un triomphe par substitution éclate dans ce que l'on appelle, après les Américains, le " politiquement correct ». La politique a perdu toute autonomie, vidée de son contenu, parce que l'éthique s'est substituée à elle en laissant croire, et là réside sa ruse ultime en forme de supercherie, qu'il s'agit encore de politique. En réalité, il conviendrait mieux de parler du « moralement correct ». Or, ce qui est moral ne fait pas nécessairement une bonne politique. Ce qui est politique ne suit pas toujours les voies de l'éthique. Il ne suffit pas d'être anti-raciste pour définir une bonne politique de l'immigration. Quand le roi Louis IX, en qui l'Eglise voit pourtant le modèle du monarche chrétien, réserva à Paris un quartier pour les prostituées, il savait bien qu'il ne faisait pas là un acte proprement moral, mais il le jugeait indispensable au bien de la Cité. Pour reprendre notre image du début, si l'éthique et la politique sont bien issues du bien et de la nécessité, elles ne combinent pas ce même double héritage dans des proportions identiques.

Il est bon, je crois de nous redire cela alors que s'impose un peu partout une éthique sécularisée qui ne se préoccupe plus de ses fondements et tient à distance respectueuse toute forme de transcendance, religieuse ou métaphysique, impose un discours convenu, de nature essentiellement morale, en recourant à la force publique. Or, il n'existe pas de liberté à l'ombre d'un quelconque terrorisme de la pensée.

Le vieux professeur de théologie morale dont jévoquais le souvenir au début de cette conférence verrait sa prédiction aujourd'hui réalisée, quand il annonçait que cette discipline serait un jour enviée des autres. Il n'avait pas prévu toutefois que ce succès allait être si éclatant ni si écrasant. Il n'avait pas prévu que, malade de son triomphe, l'éthique risquait d'y perdre son âme.

\section{Le Genie du Christianisme}

Que faire alors? Que faire quand le "politiquement correct " prend en otage l'histoire des peuples et retire aux spécialistes, je veux dire aux historiens patentés, le droit de se prononcer de manière inhabituelle sur des faits dont la mémoire a été « sanctuarisée » ? Que faire quand un certain nombre d'opinions font désormais l'objet d'un délit, je songe en particulier à l'avortement, à la différence sexuelle ou au «mariage gay », ou encore à l'existence d'une vérité absolue ? Que faire quand la loi 
sanctionne toute critique faite au judaïsme, immédiatement qualifiée d'antisémitisme, ou à l'islam, afin d'éradiquer toute forme d'islamophobie, tandis que le christianisme, lui, peut faire l'objet des critiques et des caricatures les plus féroces ? Que faire quand l'éthique dont se réclament les plus hautes institutions internationales, à commencer par les européennes, prennent ouvertement le contre-pied de la morale catholique ? Il ne devrait échapper à personne que dans plusieurs pays, comme le Québec, la France, la Belgique ou même l'Espagne, c'est le christianisme lui-même qui risque, à terme, de devenir illégal. N'a-t-on pas demandé à des écoles catholiques de ne plus se référer à ce qui, dans le Catéchisme ou même la Bible, allait à l'encontre des idées dominantes du moment?

Après la Révolution française, alors que l'Eglise avait été ruinée dans ses plus grandes profondeurs, un écrivain célèbre, Chateaubriand, osa écrire «Le Génie du christianisme », un livre qui connut le plus grand succès populaire. N'est-il pas temps d'illustrer encore aujourd'hui ce génie de notre religion? N'est-ce pas le rôle qui échoit particulièrement à ces institutions d'Eglise chargées de faire dialoguer la foi et la raison humaine, aux Universités, à vous donc ? Qu'il me soit permis de faire trois suggestions dans cette dernière partie.

a) Comme nous venons de le voir, l'éthique a rusé en gonflant trois concepts : la démocratie, la responsabilité et les droits de l'homme. Redisons donc que la démocratie est une technique propre au domaine politique auquel elle doit être restituée. Le danger est aujourd'hui d'en faire une valeur morale et de la choisir comme technique d'élaboration des normes éthiques. C'est exactement le propos avoué des "éthiques procédurales » qui font l'objet d'une discussion serrée dans l'encyclique Veritatis splendor du bienheureux Jean-Paul II. Publiée en I993, ce texte restera un des plus importants du pontificat. Puisque, dans une société sécularisée et marquée par le pluralisme culturel, il est devenu impossible de fonder ces normes sur des bases religieuses ou métaphysiques, retenons ce que nous avons de meilleur, la démocratie, et utilisons-la comme procédure afin de parvenir à fixer les normes dont la société a besoin. Leéthique procédurale donne lucidement congé au bien moral : elle sait qu'elle ne fait faire davantage que de proposer ce qui est « right " ou «wrong " à la société à un moment déterminé de son histoire.

b) Pas davantage, personne ne songerait à nier l'importance capitale des droits de 1'hom me, mais il convient d'ajouter immédiatement 
que l'origine, ou le fondement, de ces droits reste problématique. Viennent-ils de la société elle-même qui s'octroierait le droit de les reconnaître ou de les nier ? Ce serait lui accorder un statut proprement divin, ce contre quoi le christianisme s'est insurgé depuis ses débuts, au prix des persécutions que vous savez. César n'est pas Dieu. Les droits de l'hommes tirent-ils leur origine de la dignité de la personne humaine, proposition à laquelle la christianisme souscrit pleinement ? Il resterait à définir le concept de dignité, et nous savons bien qu'une société sécularisée ne pourra jamais admettre que cette dignité vienne d'une création à l'image de Dieu, car elle cesserait du coup d'être sécularisée.

Puis-je me référer à un souvenir personnel ? Le gouvernement anglais avait proposé que quatre membres de la Curie romaine puissent accompagner le pape Benoît XVI dans son voyage en Grande-Bretagne ; jétais de ceux-là. Il serait faible de parler de succès : nous avons vécu pendant cinq jours une sorte de retournement de l'Histoire. Une image suffira. C'était dans la magnifique salle gothique de Westminster Hall. Là, se trouvait rassemblé tout ce que le pays comptait d'élites de la vie politique - notamment les parlementaires - et de la société civile : une assistance nombreuse, polie et curieuse, anglaise en un mot. Annoncé par les trompettes d'argent qui ne sonnent habituellement que pour la reine, s'est avancé, non sans une certaine timidité, un homme âgé et vêtu de blanc et rouge (qui sont, comme vous le savez, les couleurs de la Résurrection). D'une voix basse et parfois lasse, il a prononcé un discours qui marquera le pontificat de Benoît XVI, mais aussi les esprits de ceux qui étaient présents au point de l'applaudir avec une chaleur inattendue - en rien anglaise - pendant de très longues minutes!

De quoi avait parlé le Pape ? Après avoir rappelé que le Parlement britannique faisait l'admiration de tous ceux qui, comme les catholiques, étaient attachés à la poursuite du bien commun, à la vie démocratique et au respect des libertés personnelles, il a noté que la question fondamentale en ce début du XXIe siècle était une question éternelle, se posant à chaque génération : sur quoi s'appuient les lois ? Au nom de quoi les gouvernements peuvent-ils imposer des contraintes aux citoyens? Si finalement tout phénomène politique trouve en son origine une question morale, quels sont, en fin de compte, les fondements éthiques du discours civil ? Les réponses pragmatiques s'avèrent là trop courtes et trop incertaines. Si la loi repose sur un simple consensus 
social, comme le prétendent les "éthiques procédurales ", elle s'expose à une grande fragilité, tant nous savons les opinions vulnérables aux modes volatiles et aux émotions passagères.

On n'a pas besoin d'être chrétien pour devenir un bon artisan du bien commun : l'Histoire est pleine de témoignages donnés par des « hommes de bonne volonté » qui ne croyaient ni au Christ, ni même en Dieu. « Les normes objectives qui dirigent une action droite, rappelait le Pape après les théologiens les plus classiques, sont accessibles à la raison, sans le contenu de la Révélation ». En ce sens, il n'existe pas de politique chrétienne, ni, comme le voulait Bossuet, de politique tirée de l'Ecriture sainte. Mais, laissée à elle-même, la raison reste exposée à des risques et des distorsions.

c) Reste la responsabilité. C'est sans doute l'une des grâces de notre temps que d'avoir perçu la responsabilité sous la forme lancinante d'une obsession. Avec des philosophes comme Emmanuel Lévinas, par exemple, elle est devenue l'autre nom de l'éthique moderne. Nous serions entrés selon l'heureuse expression d'un sociologue dans "Le temps des responsables ». Les génocides répétés, les guerres les plus meurtrières de l'histoire des hommes, et l'horreur absolue des camps nazis et soviétiques, ont fait de la responsabilité un objet tellement encombrant que nous serions tentés, en ce début de millénaire, de nous en défaire sur le bord du chemin. Impossible pourtant : elle réapparaît toujours avec l'opiniâtreté indifférente des évidences. Rien ni personne ne parvient à faire taire cette petite voix : «Qu'as-tu fait de moi ? » Qui est ce moi ? Moi, c'est moi, bien sûr, mais c'est aussi l'autre. Il s'agit de répondre présent, de répondre à la question par une présence : « $\mathrm{Me}$ voici ». Le contenu de la responsabilité, c'est donc cette présence à soi et aux autres.

Il me semble que le christianisme peut apporter ici une note décisive. Nous voyons bien que le risque d'une société sécularisée est de s'en tenir à une vision sèche et rigide de la responsabilité. Quand un drame éclate quelque part, le réflexe premier est de se demander : où sont les responsables? Que l'on réclame des comptes, que l'on juge des coupables, que l'on impose des réparations relève tout simplement du devoir de justice. Il n'y a rien à redire en cela. Mais qu'est-ce qu'une justice qui ne serait que juste ? La morale évangélique voit dans le pardon la forme la plus élevée de la justice, son assomption, en somme. Voici donc un «incontournable »: montrer qu'une société qui ne pratiquerait que les règles de la stricte justice serait condamnée à une sévérité 
insupportable. On ne saurait vivre sans pardon. Son absence éventuelle dans la vie d'un couple fait qu'une crise devient vite insupportable : ou les conjoints se pardonnent l'un l'autre, ou ils se séparent. Il en va de même dans la vie personnelle : si je suis incapable de me pardonner à moi-même, je ne puis accepter les autres. Il en va encore de même dans toute vie sociale. Voilà donc un domaine dans lequel l'Eglise peut délivrer un message unique, proprement libérateur : le pardon désigne cette alchimie mystérieuse qui transforme le mal occasionné et subi en nouvelle chance. Le pardon est la condition de l'espérance, ainsi que le redisait le pape Benoît XVI dans son encyclique Spe salvit.

1 Conférence donnée à Cracovie, le I6 octobre 20I3, à l'Université Jean-Paul II. [Conference proclaimed in The Pontifical University of John Paul II in Krakow, October 16,2013$]$. 